\title{
Some Bounds for the Polar Derivative of a Polynomial
}

\author{
Jiraphorn Somsuwan' ${ }^{1}$ and Keaitsuda Maneeruk Nakprasit $\mathbb{D}^{2}$ \\ ${ }^{1}$ Department of Mathematics, Faculty of Science, Nakhon Phanom University, Nakhon Phanom 48000, Thailand \\ ${ }^{2}$ Department of Mathematics, Faculty of Science, Khon Kaen University, Khon Kaen 40002, Thailand
}

Correspondence should be addressed to Keaitsuda Maneeruk Nakprasit; kmaneeruk@hotmail.com

Received 29 September 2017; Revised 12 January 2018; Accepted 30 January 2018; Published 1 March 2018

Academic Editor: Narendra K. Govil

Copyright (c) 2018 Jiraphorn Somsuwan and Keaitsuda Maneeruk Nakprasit. This is an open access article distributed under the Creative Commons Attribution License, which permits unrestricted use, distribution, and reproduction in any medium, provided the original work is properly cited.

\begin{abstract}
The polar derivative of a polynomial $p(z)$ of degree $n$ with respect to a complex number $\alpha$ is a polynomial $n p(z)+(\alpha-z) p^{\prime}(z)$, denoted by $D_{\alpha} p(z)$. Let $1 \leq R \leq k$. For a polynomial $p(z)$ of degree $n$ having all its zeros in $|z| \leq k$, we investigate a lower bound of modulus of $D_{\alpha} p(z)$ on $|z|=R$. Furthermore, we present an upper bound of modulus of $D_{\alpha} p(z)$ on $|z|=R$ for a polynomial $p(z)$ of degree $n$ having no zero in $|z|<k$. In particular, our results in case $R=1$ generalize some well-known inequalities.
\end{abstract}

\section{Introduction}

One of the interesting and fruitful subjects in geometry of polynomials is the geometrical relation between the modulus of a complex polynomial on a circle and the position of zeros of this polynomial inside or outside this circle. Many propositions in the area of polynomial inequalities are presented by Bernstein-type inequalities. Many results are derived from Bernstein's inequality. We start with a result due to Bernstein (see [1]). Let $p(z)$ be a polynomial of degree $n$. Then according to the well-known result known as Bernstein's inequality, we have

$$
\max _{|z|=1}\left|p^{\prime}(z)\right| \leq n \max _{|z|=1}|p(z)| .
$$

The equality holds if and only if $p(z)$ has all its zeros at the origin.

For the class of polynomials having no zero in $|z|<1$, Lax [2] proved that if $p(z)$ is a polynomial of degree $n$ having no zero in $|z|<1$, then

$$
\max _{|z|=1}\left|p^{\prime}(z)\right| \leq \frac{n}{2} \max _{|z|=1}|p(z)|
$$

The result is best possible and equality holds for $p(z)=\lambda+$ $\mu z^{n},|\lambda|=|\mu|$.
On the other hand, Turán [3] showed that, for a polynomial having all its zeros in $|z| \leq 1$,

$$
\max _{|z|=1}\left|p^{\prime}(z)\right| \geq \frac{n}{2} \max _{|z|=1}|p(z)| \text {. }
$$

Inequality (3) is best possible and becomes equality for polynomials which have all its zeros on $|z|=1$.

An extension of inequality (3) was shown by Govil [4] that, for a polynomial $p(z)$ of degree $n$ having all its zeros in $|z| \leq k, k \geq 1$,

$$
\max _{|z|=1}\left|p^{\prime}(z)\right| \geq \frac{n}{1+k^{n}} \max _{|z|=1}|p(z)| .
$$

Inequality (4) is best possible and equality holds for a polynomial $p(z)=z^{n}+k^{n}$.

In 1999, Dewan et al. [5] proved the following result.

Theorem 1 (see [5]). Let $p(z)=a_{0}+\sum_{v=\mu}^{n} a_{v} z^{\nu}, 1 \leq \mu \leq n$, be a polynomial of degree $n$ such that $p(z)$ has no zero in $|z|<k$, $k>0$. Then, for $0<r \leq R \leq k$,

$$
\begin{aligned}
\max _{|z|=r}|p(z)| \geq & \left(\frac{k^{\mu}+r^{\mu}}{k^{\mu}+R^{\mu}}\right)^{n / \mu} \max _{|z|=R}|p(z)| \\
& +\left[1-\left(\frac{k^{\mu}+r^{\mu}}{k^{\mu}+R^{\mu}}\right)^{n / \mu}\right] \min _{|z|=k}|p(z)| .
\end{aligned}
$$


Inequality (5) is best possible and becomes equality for a polynomial $p(z)=\left(z^{\mu}+k^{\mu}\right)^{n / \mu}$, where $n$ is multiple of $\mu$.

The polar derivative of a polynomial $p(z)$ of degree $n$ with respect to a complex number $\alpha$, denoted by $D_{\alpha} p(z)$, is defined by

$$
D_{\alpha} p(z)=n p(z)+(\alpha-z) p^{\prime}(z) \text {. }
$$

The polynomial $D_{\alpha} p(z)$ is of degree at most $n-1$ and it generalizes the ordinary derivative in the sense that $\lim _{\alpha \rightarrow \infty}\left(D_{\alpha} p(z) / \alpha\right)=p^{\prime}(z)$.

In 1998, Aziz and Rather [6] established the extension of inequality (4) to a polar derivative of a polynomial.

Theorem 2 (see [6]). Let $p(z)$ be a polynomial of degree $n$ having all its zeros in $|z| \leq k, k \geq 1$.

Then, for every real or complex number $\alpha$ with $|\alpha| \geq k$,

$$
\max _{|z|=1}\left|D_{\alpha} p(z)\right| \geq \frac{n(|\alpha|-k)}{1+k^{n}} \max _{|z|=1}|p(z)| .
$$

In 2008, Dewan and Upadhye [7] proved the following theorem.

Theorem 3 (see [7]). Let $p(z)$ be a polynomial of degree $n$ having all its zeros in $|z| \leq k, k \geq 1$.

Then, for every real or complex number $\alpha$ with $|\alpha| \geq k$,

$$
\begin{aligned}
& \max _{|z|=1}\left|D_{\alpha} p(z)\right| \geq n(|\alpha|-k) \\
& \quad \cdot\left[\frac{1}{1+k^{n}} \max _{|z|=1}|p(z)|+\frac{1}{2 k^{n}}\left(\frac{k^{n}-1}{k^{n}+1}\right) \min _{|z|=k}|p(z)|\right] .
\end{aligned}
$$

Furthermore, Dewan et al. [8] proved the following result.

Theorem 4 (see [8]). If $p(z)=a_{0}+\sum_{v=\mu}^{n} a_{v} z^{\nu}, 1 \leq \mu \leq n$, is a polynomial of degree $n$ having no zero in $|z|<k, k \geq 1$, then, for every real or complex number $\alpha$ with $|\alpha| \geq 1$,

$$
\begin{aligned}
& \max _{|z|=1}\left|D_{\alpha} p(z)\right| \\
& \quad \leq \frac{n}{1+s_{0}}\left[\left(|\alpha|+s_{0}\right) \max _{|z|=1}|p(z)|-(|\alpha|-1) m\right],
\end{aligned}
$$

where $m=\min _{|z|=k}|p(z)|$ and $s_{0}=k^{\mu+1}\left[\left((\mu / n)\left(\left|a_{\mu}\right| /\left(\left|a_{0}\right|-\right.\right.\right.\right.$ $\left.\left.m)) k^{\mu-1}+1\right) /\left((\mu / n)\left(\left|a_{\mu}\right| /\left(\left|a_{0}\right|-m\right)\right) k^{\mu+1}+1\right)\right]$.

Let $1 \leq R \leq k$. In this paper, we investigate a lower bound of $\left|D_{\alpha} p(z)\right|$ on $|z|=R$ for a polynomial $p(z)$ of degree $n$ having all its zeros in $|z| \leq k$ and an upper bound of $\left|D_{\alpha} p(z)\right|$ on $|z|=R$ for a polynomial $p(z)$ of degree $n$ having no zero in $|z|<k$.

\section{Main Results}

Theorem 5. Let $p(z)=a_{0}+\sum_{\nu=\mu}^{n} a_{v} z^{\nu}, 1 \leq \mu \leq n, a_{0} \neq 0$, be a polynomial of degree $n$ having all its zeros in $|z| \leq k, k \geq 1$.
Then, for every real or complex number $\alpha$ with $|\alpha| \geq k$ and $1 \leq R \leq k$,

$$
\begin{aligned}
\max _{|z|=R}\left|D_{\alpha} p(z)\right| \geq n R^{n-1}(|\alpha|-k) \\
\cdot\left[\frac{R^{n}}{R^{n}+k^{n}}\left(\frac{k^{\mu}+1}{k^{\mu}+R^{\mu}}\right)^{n / \mu} \max _{|z|=1}|p(z)|\right. \\
+\left(\frac{k^{n}}{R^{n}+k^{n}}\left(1-\left(\frac{k^{\mu}+1}{k^{\mu}+R^{\mu}}\right)^{n / \mu}\right)\right. \\
\left.\left.+\frac{1}{2 k^{n}}\left(\frac{k^{n}-R^{n}}{k^{n}+R^{n}}\right)\right) m\right],
\end{aligned}
$$

where $m=\min _{|z|=k}|p(z)|$.

Proof. Let $F(z)=p(R z)$. Observe that $F(z)$ has all its zeros in $|z| \leq k / R$.

Applying Theorem 3 to $F(z)$, we obtain that

$$
\begin{aligned}
& \max _{|z|=1}\left|D_{\alpha / R} F(z)\right| \geq n\left(\frac{|\alpha|-k}{R}\right)\left[\frac{R^{n}}{R^{n}+k^{n}} \max _{|z|=1}|F(z)|\right. \\
& \left.\quad+\frac{R^{n}}{2 k^{n}}\left(\frac{k^{n}-R^{n}}{k^{n}+R^{n}}\right) \min _{|z|=k / R}|F(z)|\right] .
\end{aligned}
$$

Applying the relations $\max _{|z|=1}\left|D_{\alpha / R} F(z)\right|=\max _{|z|=R}\left|D_{\alpha} p(z)\right|$, $\max _{|z|=1}|F(z)|=\max _{|z|=R}|p(z)|$, and $\min _{|z|=k / R}|F(z)|=$ $\min _{|z|=k}|p(z)|$ into inequality (11), we have that

$$
\begin{aligned}
& \max _{|z|=R}\left|D_{\alpha} p(z)\right| \geq n R^{n-1}(|\alpha|-k)\left[\frac{1}{R^{n}+k^{n}} \max _{|z|=R}|p(z)|\right. \\
& \left.\quad+\frac{1}{2 k^{n}}\left(\frac{k^{n}-R^{n}}{k^{n}+R^{n}}\right) \min _{|z|=k}|p(z)|\right] .
\end{aligned}
$$

Let $q(z)=z^{n} \overline{p(1 / \bar{z})}=\overline{a_{0}} z^{n}+\sum_{\nu=\mu}^{n} \overline{a_{\nu}} z^{n-\nu}$.

Since $p(z)$ has all its zeros in $|z| \leq k, q(z)$ has no zero in $|z|<1 / k$.

Let $Q(z)=q\left(z /\left(k^{2}\right)\right)$. Then $Q(z)$ is a polynomial of degree $n$ having no zero in $|z|<k$. Applying Theorem 1 to $Q(z)$ with $r=1$, we obtain that

$$
\begin{aligned}
\max _{|z|=1}|Q(z)| \geq & \left(\frac{k^{\mu}+1}{k^{\mu}+R^{\mu}}\right)^{n / \mu} \max _{|z|=R}|Q(z)| \\
& +\left(1-\left(\frac{k^{\mu}+1}{k^{\mu}+R^{\mu}}\right)^{n / \mu}\right) \min _{|z|=k}|Q(z)| .
\end{aligned}
$$

By maximum modulus principle (see [9], p. 128), we obtain that

$$
\begin{aligned}
& \begin{aligned}
\max _{|z|=1}|Q(z)| & =\frac{1}{k^{2 n}} \max _{|z|=1}\left|\overline{p\left(\frac{k^{2}}{\bar{z}}\right)}\right| \\
& \leq \frac{1}{k^{2 n}} \max _{|z|=k^{2}} \overline{\left|p\left(\frac{k^{2}}{\bar{z}}\right)\right|} \leq \frac{1}{k^{2 n}} \max _{|z|=R}|p(z)|, \\
\max _{|z|=R}|Q(z)| & =\frac{R^{n}}{k^{2 n}} \max _{|z|=R}\left|\overline{p\left(\frac{k^{2}}{\bar{z}}\right)}\right| \geq \frac{R^{n}}{k^{2 n}} \max _{|z|=1}|p(z)|,
\end{aligned} \\
& \text { and } \min _{|z|=k}|Q(z)|=\left(1 / k^{n}\right) \min _{|z|=k}|p(z)| .
\end{aligned}
$$



that

Combining these relations with inequality (13), we obtain

$$
\begin{aligned}
\max _{|z|=R}|p(z)| \geq & \left(\frac{k^{\mu}+1}{k^{\mu}+R^{\mu}}\right)^{n / \mu} R^{n} \max _{|z|=1}|p(z)| \\
& +\left(1-\left(\frac{k^{\mu}+1}{k^{\mu}+R^{\mu}}\right)^{n / \mu}\right) k^{n} \min _{|z|=k}|p(z)| .
\end{aligned}
$$

This inequality together with inequality (12) yields

$$
\begin{aligned}
& \max _{|z|=R}\left|D_{\alpha} p(z)\right| \geq n R^{n-1}(|\alpha|-k) \\
& \cdot\left[\frac{R^{n}}{R^{n}+k^{n}}\left(\frac{k^{\mu}+1}{k^{\mu}+R^{\mu}}\right)^{n / \mu} \max _{|z|=1}|p(z)|\right. \\
& +\left(\frac{k^{n}}{R^{n}+k^{n}}\left(1-\left(\frac{k^{\mu}+1}{k^{\mu}+R^{\mu}}\right)^{n / \mu}\right)\right. \\
& \left.\left.+\frac{1}{2 k^{n}}\left(\frac{k^{n}-R^{n}}{k^{n}+R^{n}}\right)\right) m\right]
\end{aligned}
$$

where $m=\min _{|z|=k}|p(z)|$.

Remark 6. Inequality (10) is best possible and the equality holds for $p(z)=z-k$ when $R=1$ and $\alpha \geq k \geq 1$ or $R=n \geq 2$ and $\alpha \geq k=\sqrt{n}$.

Remark 7. In case $R=1$, inequality (10) reduces to

$$
\begin{aligned}
& \max _{|z|=1}\left|D_{\alpha} p(z)\right| \geq n(|\alpha|-k)\left[\frac{1}{1+k^{n}} \max _{|z|=1}|p(z)|\right. \\
& \left.+\frac{1}{2 k^{n}}\left(\frac{k^{n}-1}{k^{n}+1}\right) \min _{|z|=k}|p(z)|\right] .
\end{aligned}
$$

This means that Theorem 5 generalizes Theorem 3 .

Theorem 8. If $p(z)=a_{0}+\sum_{\nu=\mu}^{n} a_{\nu} z^{\nu}, 1 \leq \mu \leq n$, is a polynomial of degree $n$ having no zero in $|z|<k, k \geq 1$, then, for every real or complex number $\alpha$ with $|\alpha| \geq R$ and $0<r \leq R \leq k$,

$$
\begin{gathered}
\max _{|z|=R}\left|D_{\alpha} p(z)\right| \leq \frac{n}{1+s_{0}}\left[\left(\frac{|\alpha|}{R}+s_{0}\right)\left(\frac{k^{\mu}+R^{\mu}}{k^{\mu}+r^{\mu}}\right)^{n / \mu}\right. \\
\cdot \max _{|z|=r}|p(z)|-\left[\left(\frac{|\alpha|}{R}-1\right)+\left(\frac{|\alpha|}{R}+s_{0}\right)\right. \\
\left.\left.\cdot\left(\frac{k^{\mu}+R^{\mu}}{k^{\mu}+r^{\mu}}\right)^{n / \mu}\left(1-\left(\frac{k^{\mu}+r^{\mu}}{k^{\mu}+R^{\mu}}\right)^{n / \mu}\right)\right] m\right],
\end{gathered}
$$

where $m=\min _{|z|=k}|p(z)|$ and $s_{0}=(k / R)^{\mu+1}\left[\left((\mu / n)\left(\left|R^{\mu} a_{\mu}\right| /\right.\right.\right.$ $\left.\left.\left.\left(\left|a_{0}\right|-m\right)\right)(k / R)^{\mu-1}+1\right) /\left((\mu / n)\left(\left|R^{\mu} a_{\mu}\right| /\left(\left|a_{0}\right|-m\right)\right)(k / R)^{\mu+1}+1\right)\right]$.

The equality holds for a polynomial $p(z)=z+k$, where $\alpha \in \mathbb{R}$ with $\alpha \geq 1$.

Proof. Let $F(z)=p(R z)$. Observe that $F(z)$ has no zero in $|z|<k / R$.
Applying Theorem 4 to $F(z)$, we obtain that

$$
\begin{aligned}
& \max _{|z|=1}\left|D_{\alpha / R} F(z)\right| \leq \frac{n}{1+s_{0}}\left[\left(\frac{|\alpha|}{R}+s_{0}\right) \max _{|z|=1}|F(z)|\right. \\
& \left.-\left(\frac{|\alpha|}{R}-1\right) \min _{|z|=k / R}|F(z)|\right],
\end{aligned}
$$

where $s_{0} \quad=\quad(k / R)^{\mu+1}\left[\left((\mu / n)\left(\left|R^{\mu} a_{\mu}\right| /\left(\left|a_{0}\right| \quad-\right.\right.\right.\right.$ $\left.\left.\left.\min _{|z|=k / R}|F(z)|\right)\right)(k / R)^{\mu-1}+1\right) /\left((\mu / n)\left(\left|R^{\mu} a_{\mu}\right| /\left(\left|a_{0}\right| \quad-\right.\right.\right.$ $\left.\left.\left.\left.\min _{|z|=k / R}|F(z)|\right)\right)(k / R)^{\mu+1}+1\right)\right]$.

We apply the relations $\max _{|z|=1}\left|D_{\alpha / R} F(z)\right|=$ $\max _{|z|=R}\left|D_{\alpha} p(z)\right|, \max _{|z|=1}|F(z)|=\max _{|z|=R}|p(z)|$, and $\min _{|z|=k / R}|F(z)|=\min _{|z|=k}|p(z)|$ into inequality (19) to obtain

$$
\begin{aligned}
& \max _{|z|=R}\left|D_{\alpha} p(z)\right| \\
& \quad \leq \frac{n}{1+s_{0}}\left[\left(\frac{|\alpha|}{R}+s_{0}\right) \max _{|z|=R}|p(z)|-\left(\frac{|\alpha|}{R}-1\right) m\right],
\end{aligned}
$$

where $m=\min _{|z|=k}|p(z)|$ and $s_{0}=(k / R)^{\mu+1}\left[\left((\mu / n)\left(\left|R^{\mu} a_{\mu}\right| /\right.\right.\right.$ $\left.\left.\left.\left(\left|a_{0}\right|-m\right)\right)(k / R)^{\mu-1}+1\right) /\left((\mu / n)\left(\left|R^{\mu} a_{\mu}\right| /\left(\left|a_{0}\right|-m\right)\right)(k / R)^{\mu+1}+1\right)\right]$.

Theorem 1 implies that

$$
\begin{aligned}
\max _{|z|=R}|p(z)| \leq & \left(\frac{k^{\mu}+R^{\mu}}{k^{\mu}+r^{\mu}}\right)^{n / \mu} \max _{|z|=r}|p(z)| \\
& -\left(\left(\frac{k^{\mu}+R^{\mu}}{k^{\mu}+r^{\mu}}\right)^{n / \mu}-1\right) \min _{|z|=k}|p(z)| .
\end{aligned}
$$

This inequality together with inequality (20) yields

$$
\begin{aligned}
& \max _{|z|=R}\left|D_{\alpha} p(z)\right| \leq \frac{n}{1+s_{0}}\left[\left(\frac{|\alpha|}{R}+s_{0}\right)\left(\frac{k^{\mu}+R^{\mu}}{k^{\mu}+r^{\mu}}\right)^{n / \mu}\right. \\
& \cdot \max _{|z|=r}|p(z)|-\left[\left(\frac{|\alpha|}{R}+s_{0}\right)\left(\left(\frac{k^{\mu}+R^{\mu}}{k^{\mu}+r^{\mu}}\right)^{n / \mu}-1\right)\right. \\
& \left.\left.+\left(\frac{|\alpha|}{R}-1\right)\right] m\right],
\end{aligned}
$$

where $m=\min _{|z|=k}|p(z)|$ and $s_{0}=(k / R)^{\mu+1}\left[\left((\mu / n)\left(\left|R^{\mu} a_{\mu}\right| /\right.\right.\right.$ $\left.\left.\left.\left(\left|a_{0}\right|-m\right)\right)(k / R)^{\mu-1}+1\right) /\left((\mu / n)\left(\left|R^{\mu} a_{\mu}\right| /\left(\left|a_{0}\right|-m\right)\right)(k / R)^{\mu+1}+1\right)\right]$.

Next, we show that the equality holds for a polynomial $p(z)=z+k$, where $\alpha \in \mathbb{R}$ with $\alpha \geq 1$. Let $\alpha \in \mathbb{R}$ with $\alpha \geq 1$. Consider $p(z)=z+k$; we obtain that $\max _{|z|=R}\left|D_{\alpha} p(z)\right|=$ $|\alpha+k|$. Observe that the right-hand side of inequality (18) becomes $|\alpha|+k$. Furthermore, $|\alpha+k|=|\alpha|+k$ by condition of $\alpha$. Therefore, the equality holds for a polynomial $p(z)=$ $z+k$.

Remark 9. In case $r=R=1$, inequality (18) reduces to

$$
\begin{aligned}
\max _{|z|=1} & \left|D_{\alpha} p(z)\right| \\
& \leq \frac{n}{1+s_{0}}\left[\left(|\alpha|+s_{0}\right) \max _{|z|=1}|p(z)|-(|\alpha|-1) m\right],
\end{aligned}
$$


where $m=\min _{|z|=k}|p(z)|$ and $s_{0}=k^{\mu+1}\left[\left((\mu / n)\left(\left|a_{\mu}\right| /\left(\left|a_{0}\right|-\right.\right.\right.\right.$ $\left.\left.m)) k^{\mu-1}+1\right) /\left((\mu / n)\left(\left|a_{\mu}\right| /\left(\left|a_{0}\right|-m\right)\right) k^{\mu+1}+1\right)\right]$.

That is, inequality (18) generalizes inequality (9) in Theorem 4.

\section{Conflicts of Interest}

The authors declare that there are no conflicts of interest regarding the publication of this paper.

\section{Acknowledgments}

The first author is supported in part by Nakhon Phanom University. The second author is supported by National Research Council of Thailand and Khon Kaen University, Thailand (Grant no. kku fmis (580010)).

\section{References}

[1] A. C. Schaeffer, "Inequalities of A. Markoff and S. Bernstein for polynomials and related functions," Bulletin (New Series) of the American Mathematical Society, vol. 47, pp. 565-579, 1941.

[2] P. D. Lax, "Proof of a conjecture of P. Erdös on the derivative of a polynomial," Bulletin (New Series) of the American Mathematical Society, vol. 50, pp. 509-513, 1944.

[3] P. Turán, "Über die Ableitung von Polynomen," Compositio Mathematica, vol. 7, pp. 89-95, 1939.

[4] N. K. Govil, "On the derivative of a polynomial," Proceedings of the American Mathematical Society, vol. 41, pp. 543-546, 1973.

[5] K. K. Dewan, R. S. Yadav, and M. S. Pukhta, "Inequalities for a polynomial and its derivative," Mathematical Inequalities and Applications, vol. 2, no. 2, pp. 203-205, 1999.

[6] A. Aziz and N. A. Rather, "A refinement of a theorem of Paul Turán concerning polynomials," Mathematical Inequalities and Applications, vol. 1, no. 2, pp. 231-238, 1998.

[7] K. K. Dewan and C. M. Upadhye, "Inequalities for the polar derivative of a polynomial," Journal of Inequalities in Pure and Applied Mathematics, vol. 9, no. 4, article 119, 9 pages, 2008.

[8] K. K. Dewan, N. Singh, and A. Mir, "Extensions of some polynomial inequalities to the polar derivative," Journal of Mathematical Analysis and Applications, vol. 352, no. 2, pp. 807-815, 2009.

[9] J. B. Conway, Functions of One Complex Variable, vol. 11 of Graduate Texts in Mathematics, Springer-Verlag, New York, NY, USA, 2nd edition, 1978. 


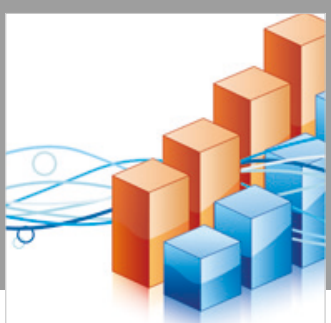

Advances in

Operations Research

\section{-n-m}
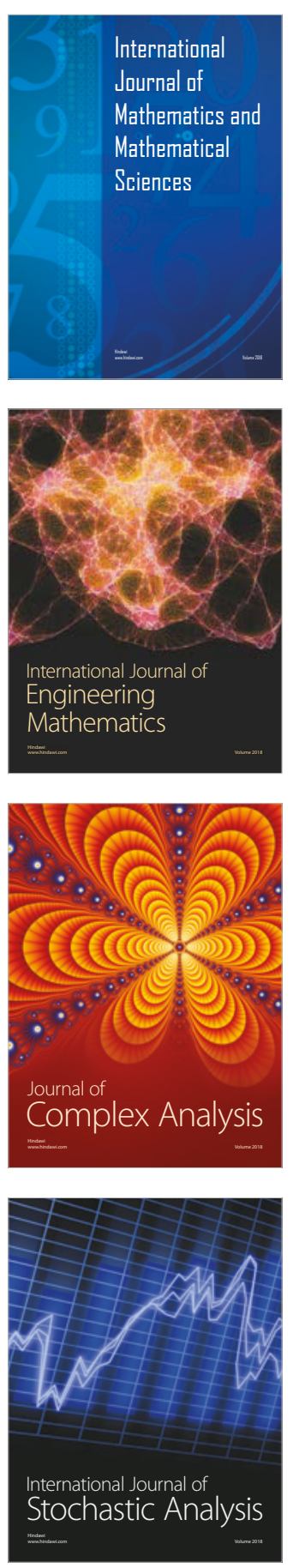
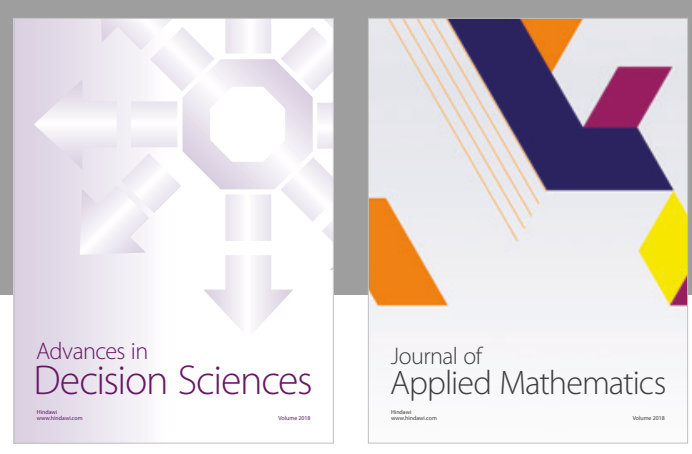

Journal of

Applied Mathematics
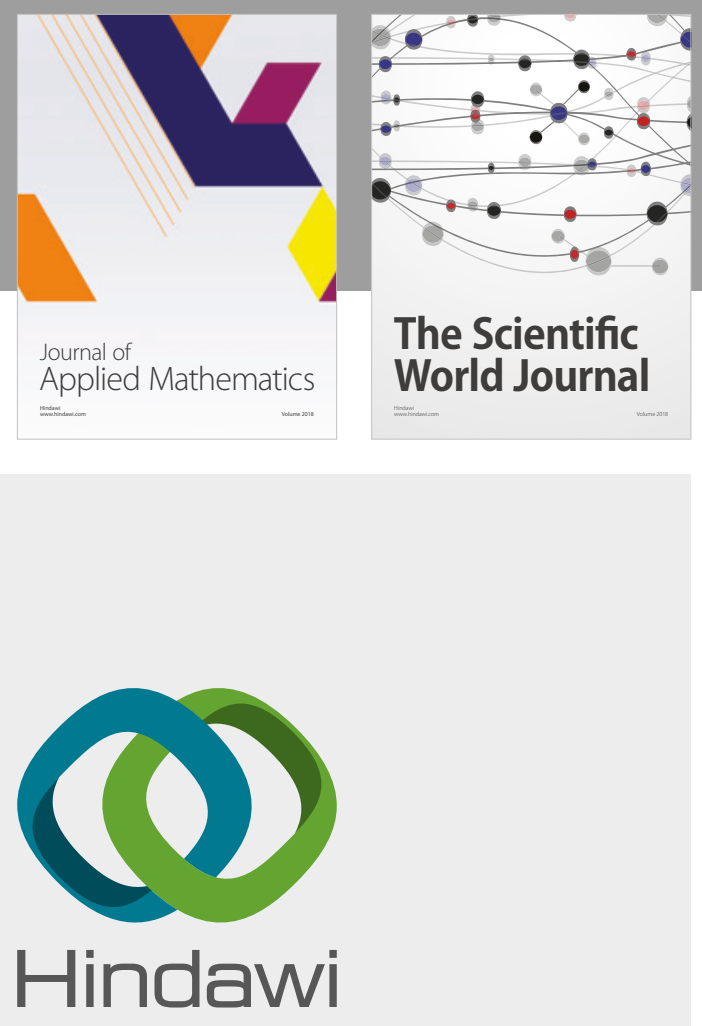

Submit your manuscripts at

www.hindawi.com

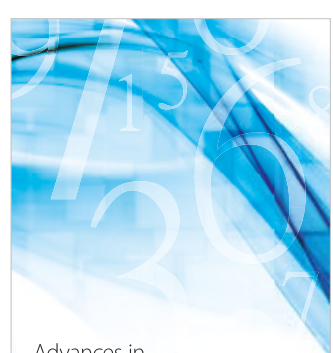

Advances in
Numerical Analysis
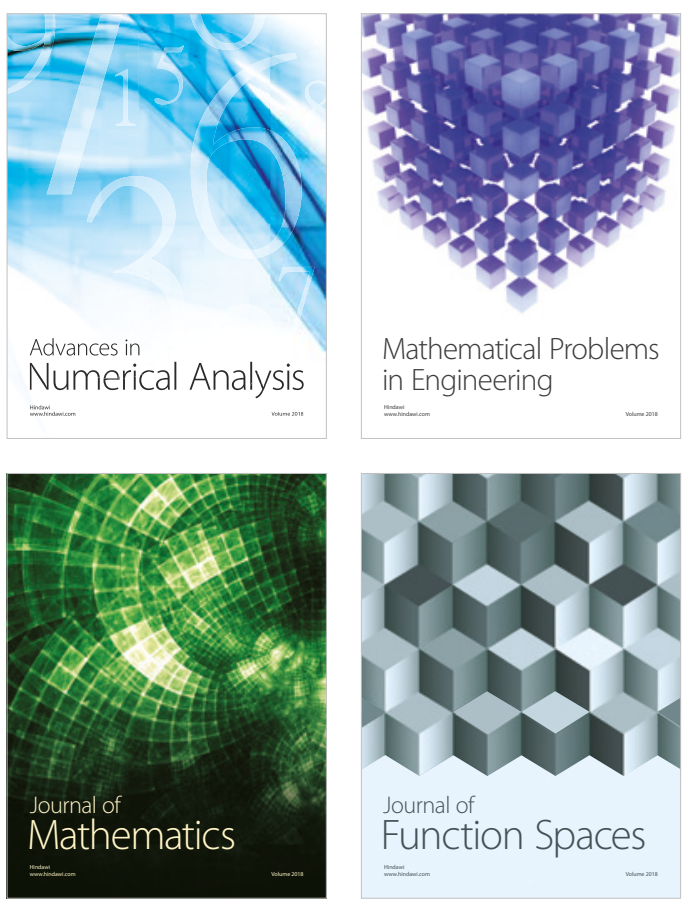

Mathematical Problems in Engineering

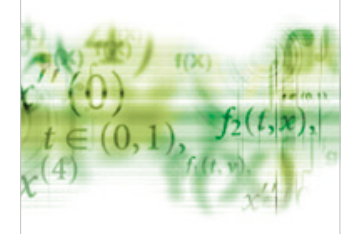

International Journal of

Differential Equations

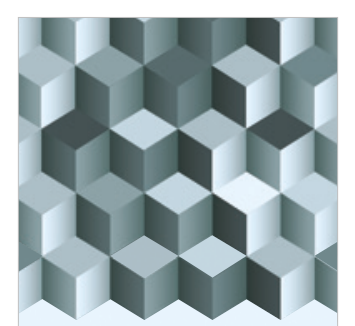

Journal of

Function Spaces

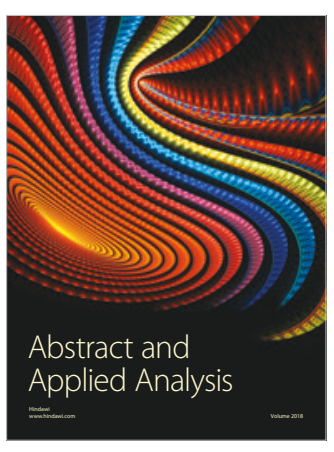

The Scientific

World Journal

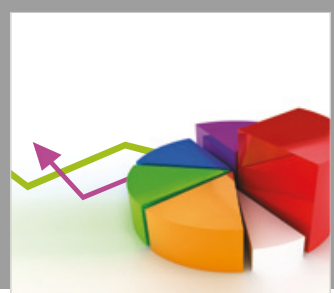

Journal of

Probability and Statistics
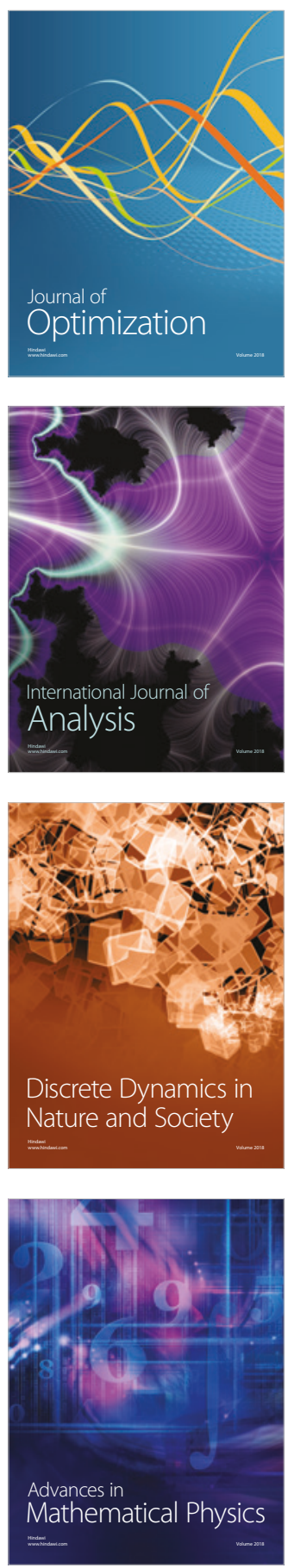